\title{
7,4'-二甲氧基洋芹素-5-O-葡萄糖苷的高效合成
}

\author{
颜世强李英霞* \\ (复旦大学药学院 上海 201203)
}

\begin{abstract}
摘要 7,4'-二甲氧基洋芹素-5-O-葡萄糖苷作为珍贵中药白木香的有效成分具有抑制 LPS 诱导巨噬细胞生成 NO 活性. 由于 5-位羟基具有较强的分子内氢键, 5-位氧苷的黄酮类化合物在传统的糖苷化条件不能高效合成. 以价廉易得的柚 皮素和 $D$-葡萄糖为原料, 经选择性羟基保护、硼氢化钠还原、相转移催化下的糖苷化、2,3-二氯-5,6-二氰基对苯醌(DDQ) 氧化等 6 步反应, 以 $36.0 \%$ 的总收率完成了 7,4'二甲氧基洋芹素-5-O-葡萄糖苗的化学合成, 为该化合物进一步的生物 活性研究奠定了物质基础.
\end{abstract}

关键词 白木香; 7,4'-二甲氧基洋芹素-5- $O$-葡萄糖苷; 黄酮; 化学合成

\section{Efficient Synthesis of 7,4'-Dimethylapigenin-5-O-glycoside}

\author{
Yan, Shiqiang Li, Yingxia* \\ (School of Pharmacy, Fudan University, Shanghai 201203)
}

\begin{abstract}
As the active component of precious Chinese medicine Aquilaria sinensis, 7,4'-dimethylapigenin-5-O-glycoside showed inhibitory activity for nitric oxide (NO) production by activated RAW 264.7 cells. Because of the strong intramolecular $\mathrm{H}$-bond, the 5-O-glucosidic linkage in flavonoids could not be efficiently constructed via conventional glycosylation method. In this paper, the efficient chemical synthesis of 7,4'-dimethylapigenin-5-O-glycoside has been achieved for the first time starting from commercially available naringenin and $D$-glucose via a linear reaction sequence of 6 steps with the overall yield of $36.0 \%$, wherein selective hydroxy protecting, reduction with sodium borohydride, glycosylation under phase transfer catalytic condition, oxidation with 2,3-dichloro-5,6-dicyano-1,4-benzoquinone (DDQ) and other reactions were used. This work definitely laid the foundation for the further pharmacological study of this natural compound.
\end{abstract}

Keywords Aquilaria sinensis; 7,4'-dimethylapigenin-5-O-glycoside; flavonoids; chemical synthesis

白木香 [Aquilaria sinensis (Lour.) Gilg]为瑞香科 (Thymelaeaceae)沉香属常绿乔木, 别名土沉香、女儿香、 莞香等. 白木香为我国特有的珍贵药源植物, 野生资源 曾经十分丰富，但长期以来由于森林资源、生态环境遭 受自然灾害和人为破坏等诸多原因, 白木香野生资源量 在不断减少, 现仅分布有零星散生的残存植株, 1999 年 白木香被国务院批准为国家二级重点保护野生植物, 现 已载入《中国植物红皮书》.

白木香产生的香脂即沉香, 是传统名贵药材和天然 香料, 味辛、苦, 性微温, 沉香具有行气止痛、温中止呕、 纳气平喘等功效. 用于治疗胸腹胀闷疼痛、胃寒呕吐呃 逆、肾虚气逆喘急有显效; 在临床上用于治疗呃逆、功 能性消化不良、痛经、前列腺痛、胃痛、胆汁反流性胃
炎等. 中成药中以沉香组方配伍的有约 200 种, 在《本 草纲目》、《本草经疏》、《大明本草》以及《本草通玄》 等药典书籍中均有记载. 现代医学试验研究表明, 白木 香具有一定的抗肿瘤 ${ }^{[1 a \sim 1 c]}$ 、抗菌 ${ }^{[2 a \sim 2 b]}$ 、镇痛抗炎 ${ }^{[3 a \sim 3 b]}$ 、 利泄 ${ }^{[4]} 、$ 降糖 ${ }^{[5]}$ 等功能.

5-位氧苷的黄酮、黄酮醇以及花青素类化合物是一 类重要的天然产物, 它们广泛存在于自然界中 ${ }^{[6 a \sim 6 f]}$. 7,4'-二甲氧基洋芹素-5- $O$ - 葡萄糖苷是北京大学屠鹏飞 课题组首次从白木香茎木中分离得到的 5-位氧苷黄酮 化合物, 生物活性测试表明其具有抑制 LPS 诱导巨噬细 胞生成 $\mathrm{NO}$ 活性, 其 $\mathrm{IC}_{50}$ 值为 $9.19 \mu \mathrm{mol} \cdot \mathrm{L}^{-1}$, 明显优于 阳性对照药布洛芬 $\left(\mathrm{IC}_{50}=94.12 \mu \mathrm{mol} \cdot \mathrm{L}^{-1}\right)^{[7]}$. 由于 $7,4^{\prime}-$ 二甲氧基洋芹素-5- $O$-葡萄糖苷在植物中含量较少, 分

\footnotetext{
* Corresponding author. E-mail: liyx417@fudan.edu.cn

Received July 23, 2018; revised August 25, 2018; published online September 10, 2018.

Project supported by the National Natural Science Foundation of China (No. 81773576).

国家自然科学基金(No. 81773576)资助项目.
} 
离提纯困难(原始文献从 $40 \mathrm{~kg}$ 的白木香茎木中仅分离 纯化得到 $23 \mathrm{mg}$ 的目标化合物), 因此限制了其在生物 活性及作用机制等方面的深入研究. 经查阅文献, 目前 尚未有该化合物的合成报道. 首次实现了 7,4'-二甲氧基 洋芹素-5- $O$ - 葡萄糖苷的化学合成, 为进一步研究其药 理活性奠定物质基础, 并为化学合成其他 5-位氧苷的黄 酮及黄酮醇类化合物及其衍生物提供参考.

\section{1 结果与讨论}

由于分子内氢键的存在, 黄酮或黄酮醇类化合物中 5-位差基糖苷键的构建一直是合成中的一个难点问题. 截止目前, 采用化学合成的方法完成黄酮或黄酮醇类化 合物 5-位氧苷的合成仅有少量的文献报道 ${ }^{[8 \mathrm{a} \sim 8 \mathrm{f}]}$, 可分为 直接法和间接法两种合成策略. 直接法的合成策略是指 糖基供体与黄酮或黄酤醇母核的 5-位酚羟基在催化剂

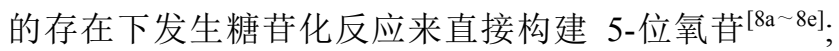
间接法的合成策略则是糖基供体与黄烷母核的 5-位酚 羟基在催化剂的存在下发生糖苷化反应得到黄烷 5-位 氧苷化合物, 然后将黄烷 5-位氧苷化合物经氧化后得到 黄酮 5-位氧苷化合物 ${ }^{[8 \mathrm{f}]}$. 本文选择利用间接合成的策略 对 7,4'-二甲氧基洋芹素-5- $O$-葡萄糖苷进行合成.

7,4'-二甲氧基洋芹素-5- $O$ - 葡萄糖苷的逆合成分析 如下(图 1): 目标化合物的制备可通过其前体黄烷糖苷 6 经 DDQ 氧化成黄酮糖苷后脱除糖环上的保护基实现. 黄烷糖苷 6 可通过关键中间体 5 与糖基供体 8 发生糖苷 化反应制备得到. 糖基供体 $\mathbf{8}$ 可通过葡萄糖经全苯甲酰 基保护后端基溴代而得，而关键中间体 $\mathbf{5}$ 则可由价廉易 得的柚皮素 2 为起始原料，通过官能团转化制备得到.

基于上述反合成分析, 以价廉易得的柚皮素 $\mathbf{2}$ 为起 始原料(图 2), 经硫酸二甲酯选择性甲基化以 $86 \%$ 的收 率得到 7,4'-二甲氧基柚皮素 3; 化合物 3 的 5-位羟基经 乙酰基保护, 使用 $95 \%$ 乙醇重结晶, 以 $96 \%$ 的收率得到 白色固体化合物 $\mathbf{4}^{[8 \mathrm{f}]}$; 二氢黄酮化合物 4 中的 4-位羰基 经硼氢化钠还原, 后处理用乙酸乙酯一石油醚重结晶以 $91 \%$ 的收率得到关键中间体黄烷化合物 $\mathbf{5}^{[8 \mathrm{f}]}$. 在此需要 指出的是, 曾尝试以 5-位羟基裸露的化合物 3 为原料进 行 4-位羰基的硼氢化钠还原, 结果反应体系变的较为复 杂, 没有得到我们期望的关键中间体黄烷化合物 5. 这 可能是由于分子内氢键造成的.

在得到关键中间体黄烷化合物 $\mathbf{5}$ 后, 本路线的另一 个关键问题即为黄烷化合物 5-位氧苷键的构建. Midori 小组 ${ }^{[\mathrm{ff}]}$ 对于黄烷化合物 5-位氧苷键的构建采用氟代糖 为糖基供体，三氟化嗍乙醚-2,6-二叔丁基-4-甲基吡啶 (DTBMP) 体系为促进剂. 采用他们的合成策略除了得 到需要的 5-位氧苷黄烷化合物以外, 还得到其重排的 6-

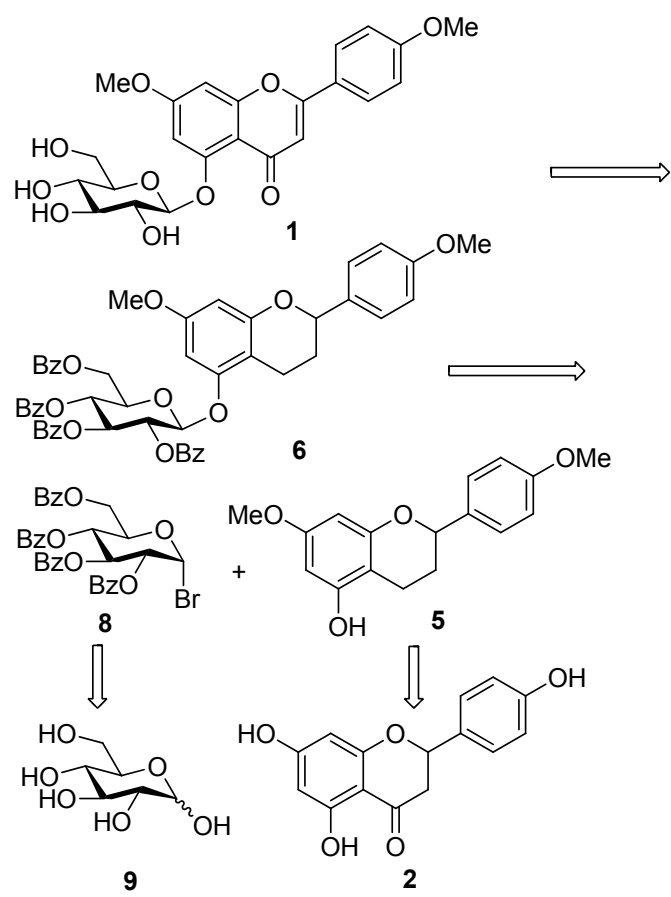

图 $17,4^{\prime}$-二甲氧基洋芹素-5- $O$-葡萄糖苷的逆合成分析 Figure 1 Retrosynthetic analysis of 7,4'-dimethylapigenin-5-O-glycoside

位碳苷黄烷化合物，且两者之间难以实现分离纯化. 虽 经过条件优化可以使重排产物所占比例降低，但最终未 能实现只得到唯一的 5-位氧苷黄烷化合物的目标. 由此 推测 Midori 小组采用酸性的三氟化嗍乙醚为糖苷化的 促进剂是引起 5-位氧苷黄烷化合物重排得到 6-位碳苷 黄烷化合物的一个重要原因.

为克服 Midori 小组的合成策略所存在的弊端，采用 溴代糖为糖基供体在碱性条件下来实现黄烷化合物 5位氧苷键的构建. $D$-葡萄糖全苯甲酰化后处理，使用 95\%乙醇重结晶以 $84 \%$ 的收率得到白色固体化合物 $\mathbf{1 0}$; 全苯甲酰化葡萄糖 $\mathbf{1 0}$ 采用乙酰溴-甲醇体系将端基溴代 以 $88 \%$ 的收率得到糖基供体 8 . 实验表明，以全苯甲酰 化的溴代葡萄糖作为糖基供体优于全乙酰化的溴代葡 萄糖. 前者室温放置数月仍未见明显分解，而后者密闭 冷藏一周左右即会出现明显的颜色变化且伴有较多杂 质的生成.

在成功制备全苯甲酰化的溴代葡萄糖糖基供体 8 后, 首先试验了相转移催化下的糖苷化反应. 在相转移 催化剂四丁基溴化铵存在的条件下，将黄烷化合物 5 与 糖基供体 8 首先室温搅拌下溶于氯仿，然后向反应体系 中加入等体积的 $0.5 \mathrm{~mol} / \mathrm{L}$ 的碳酸钾溶液 ${ }^{[9]}$. 令人高兴的 是 $45{ }^{\circ} \mathrm{C}$ 下加热反应 $12 \mathrm{~h}$ 后以 $38 \%$ 的收率得到 5-位氧 苷化合物 6, 且反应中未检测到重排产物 6-位碳苷化合 物的生成. 当采用饱和碳酸钾溶液时, $45{ }^{\circ} \mathrm{C}$ 下加热反 应 $18 \mathrm{~h}$ 后能以 $66 \%$ 的纯化后收率得到目标化合物 6 . 此 


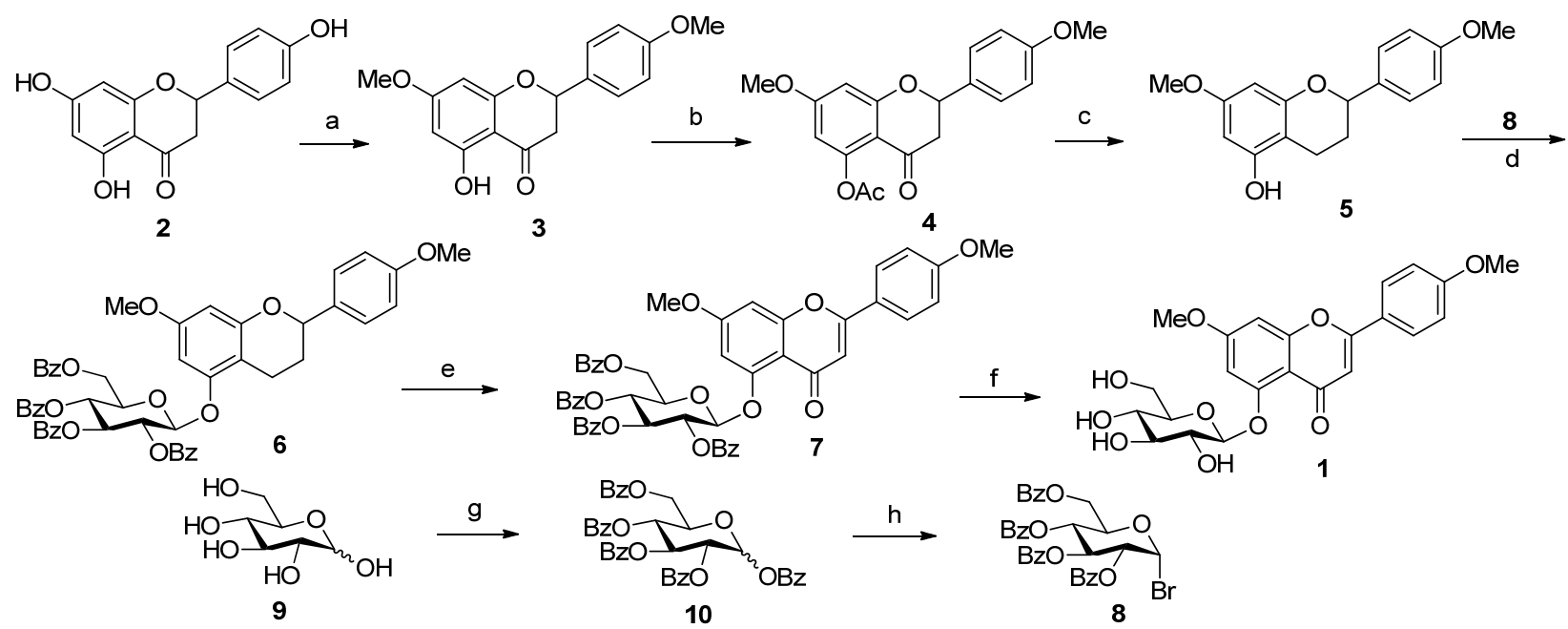

Reagents and conditions: (a) $\mathrm{Me}_{2} \mathrm{SO}_{4}$ (4.0 equiv.), $\mathrm{K}_{2} \mathrm{CO}_{3}$ (4.0 equiv.), acetone, reflux, $48 \mathrm{~h}, 86 \%$; (b) $\mathrm{Et}_{3} \mathrm{~N}$ (8.0 equiv.), DMAP (1.0 equiv.), $\mathrm{AcCl}$ (2.5 equiv.), $10 \mathrm{~min}, 96 \%$; (c) $\mathrm{NaBH}_{4}\left(6.0\right.$ equiv.), THF- $\mathrm{H}_{2} \mathrm{O}$, r.t., $3.5 \mathrm{~h}, 91 \%$; (d) TBAB (0.5 equiv.), aq. $\mathrm{K}_{2} \mathrm{CO}_{3}, 45^{\circ} \mathrm{C}, 18 \mathrm{~h}, 66 \%$; (e) DDQ (3.0 equiv.), DCM, 18 h, 78\%; (f) $\mathrm{K}_{2} \mathrm{CO}_{3}$ (2.0 equiv.), THF-MeOH-H ${ }_{2} \mathrm{O}, 2$ h, 93\%; (g) BzCl (7.5 equiv.), Py, 4 h, 84\%; (h) AcBr (5.0 equiv.), $\mathrm{MeOH}$ (3.0 equiv.), $\mathrm{AcOH}, 8 \mathrm{~h}, 88 \%$.

图 2 7,4'-二甲氧基洋芹素-5- $O$-葡萄糖苷的合成路线

Figure 2 Synthesis of 7,4'-dimethylapigenin-5-O-glycoside

外, 我们也曾尝试加热条件下，黄烷化合物 5 与糖基供 体 8 在 $N, N$-二甲基甲酰胺(DMF) 溶液中固体碳酸钾催化 下的糖苷化反应，但遗憾的是没有得到我们期望的目标 产物 ${ }^{[8 \mathrm{~d}]}$

在 DDQ 存在的条件下，黄烷糖苷化合物 6 可以被 成功地氧化以 $78 \%$ 的收率得到黄酮糖苷化合物 $7^{[8 \mathrm{f}]}$. 黄 酮糖苷化合物 7 在四氢呋喃一甲醇-水混合溶剂体系 中 $^{[10]}$, 采用 2 equiv. 的无水碳酸钾可以非常顺利地脱除 糖环上的保护基以 $93 \%$ 的收率得到需要的最终产物. 经 过比对，采用此法合成的 7,4'-二甲氧基洋芹素-5- $O$-葡 萄糖苷核磁共振图谱与天然产物数据一致 ${ }^{[7]}$.

\section{2 结论}

以价廉易得的柚皮素和 $D$-葡萄糖为原料, 经选择 性羟基保护、硼氢化钠还原、相转移催化下的糖苷化、 DDQ 氧化等 6 步反应，以 $36.0 \%$ 的总收率首次完成了 7,4'-二甲氧基洋芹素-5- $O$-葡萄糖苷的化学合成. 终产物 及中间体经 NMR 和 HRMS 结构确证. 该合成方法较之 从白木香中提取 7,4'-二甲氧基洋芹素-5- $O$-葡萄糖苷具 有原料易得、操作简单、可大量制备等优点, 为后期深 入研究其药理活性及结构改造奠定了物质基础. 同时本 研究结果进一步丰富了 5-位氧苷黄酮及黄酮醇类化合 物及其衍生物的合成方法.

\section{3 实验部分}

\section{1 仪器与试剂}

熔点采用上海精密科学仪器有限公司制造 WRR 型
熔点仪测定; 核磁共振波谱采用 Bruker VANCE-400 或 600 型核磁共振仪测定; 质谱采用 Agilent 1946BESIMS 型质谱仪测定; 旋光采用 SGW-3 自动旋光仪测定. 薄层色谱(TLC)采用 $\mathrm{HSGF}_{254}$ (烟台化学工业研究所), 柱 层析使用硅胶为 200 300 目(烟台化学工业研究所). 所 用试剂和溶剂为市售化学纯或分析纯.

\section{2 实验方法}

3.2.1 2-(4-甲氧基苯基)-5-羟基-7-甲氧基苯并二氢吡 喃-4-酮(3)的合成

将柚皮素 $2(5.00 \mathrm{~g}, 18.38 \mathrm{mmol})$ 溶于丙酮 $150 \mathrm{~mL}$, 室温摚拌下依次加入无水碳酸钾 $(10.14 \mathrm{~g}, 73.48 \mathrm{mmol})$ 及硫酸二甲酯 $(7.04 \mathrm{~mL}, 73.56 \mathrm{mmol})$. 室温摚拌 $10 \mathrm{~min}$ 后, $50{ }^{\circ} \mathrm{C}$ 下加热回流 $48 \mathrm{~h}$, 经薄层色谱(TLC) [V(石油 醚)：V(乙酸乙酯 $)=10 ： 1$ 检测显示反应完全. 过滤, 滤饼用丙酮洗涤，减压蒸除大部分的溶剂后将剩余液倾 入 $1 \mathrm{~mol} / \mathrm{L}$ 盐酸 $200 \mathrm{~mL}$ 中，二氯甲烷萃取 $(100 \mathrm{~mL} \times 2)$, 合并有机相，饱和食盐水 $100 \mathrm{~mL}$ 洗涤，无水硫酸钠干 燥. 减压浓缩柱层析得白色固体 $34.75 \mathrm{~g}$, 收率 $86 \%$. m.p. $119 \sim 120{ }^{\circ} \mathrm{C} ;{ }^{1} \mathrm{H}$ NMR (400 MHz, $\left.\mathrm{CDCl}_{3}\right) \delta: 12.04$ (s, 1H), $7.38(\mathrm{~d}, J=8.1 \mathrm{~Hz}, 2 \mathrm{H}), 6.95(\mathrm{~d}, J=8.2 \mathrm{~Hz}, 2 \mathrm{H})$, $6.06(\mathrm{~s}, 1 \mathrm{H}), 6.04(\mathrm{~s}, 1 \mathrm{H}), 5.36(\mathrm{~d}, J=12.9 \mathrm{~Hz}, 1 \mathrm{H}), 3.83$ (s, 3H), $3.80(\mathrm{~s}, 3 \mathrm{H}), 3.21 \sim 3.00(\mathrm{~m}, 1 \mathrm{H}), 2.78(\mathrm{~d}, J=17.1$ $\mathrm{Hz}, 1 \mathrm{H}) ;{ }^{13} \mathrm{C}$ NMR $\left(150 \mathrm{MHz}, \mathrm{CDCl}_{3}\right) \delta: 196.16,168.08$, $164.25,163.02,160.17,130.51,127.86,114.35,103.25$, 95.20, 94.34, 79.12, 55.79, 55.49, 43.30; HRMS calcd for $\mathrm{C}_{17} \mathrm{H}_{15} \mathrm{O}_{5} 299.0920$, found 299.0986 . 
3.2.2 2-(4-甲氧基苯基)-5-乙酰氧基-7-甲氧基苯并二 氢吡喃-4-酮(4)的合成

将化合物 3 (1.67 g, $5.56 \mathrm{mmol})$ 溶于二氯甲烷 75 $\mathrm{mL}$, 室温搅拌下依次加入三乙胺 $(6.15 \mathrm{~mL}, 44.48 \mathrm{mmol})$ 及 DMAP (0.68 g, $5.56 \mathrm{mmol})$. 向反应体系中缓慢滴加 乙酰氯 $(0.96 \mathrm{~mL}, 13.90 \mathrm{mmol}), 10 \mathrm{~min}$ 后, 经 TLC [V(石 油醚) $: V$ (乙酸乙酯 $)=6: 1]$ 检测显示反应完全. 将反应 液倾入 $1 \mathrm{~mol} / \mathrm{L}$ 盐酸 $200 \mathrm{~mL}$ 中, 二氯甲烷萃取 $(100 \mathrm{~mL} \times 2)$, 合并有机相, 饱和食盐水 $100 \mathrm{~mL}$ 洗涤, 无水硫酸钠干燥. 减压浓缩得粗品, 粗品经 $95 \%$ 乙醇重 结晶, 过滤, 干燥得白色固体 41.82 g, 收率 96\%. m.p. $160 \sim 161{ }^{\circ} \mathrm{C} ;{ }^{1} \mathrm{H}$ NMR $\left(400 \mathrm{MHz}, \mathrm{CDCl}_{3}\right) \delta: 7.37$ (d, $J=$ $8.3 \mathrm{~Hz}, 2 \mathrm{H}), 6.95$ (d, $J=8.4 \mathrm{~Hz}, 2 \mathrm{H}), 6.41$ (s, 1H), 6.28 (s, $1 \mathrm{H}), 5.40$ (d, $J=13.6 \mathrm{~Hz}, 1 \mathrm{H}), 3.83(\mathrm{~s}, 3 \mathrm{H}), 3.82(\mathrm{~s}, 3 \mathrm{H})$, 3.04 (t, $J=16.4 \mathrm{~Hz}, 1 \mathrm{H}), 2.70$ (d, $J=16.7 \mathrm{~Hz}, 1 \mathrm{H}), 2.39$ (s, $3 \mathrm{H}) ;{ }^{13} \mathrm{C} \mathrm{NMR}\left(150 \mathrm{MHz}, \mathrm{CDCl}_{3}\right) \delta: 189.22,169.71$, $165.59,164.47,160.20,151.98,130.56,127.91,114.36$, 108.07, 104.84, 99.65, 79.46, 55.94, 55.51, 44.97, 21.27; HRMS calcd for $\mathrm{C}_{19} \mathrm{H}_{18} \mathrm{NaO}_{6} 365.1001$, found 365.1021 .

3.2.3 2-(4-甲氧基苯基)-5-羟基-7-甲氧基苯并二氢吡 喃(5)的合成

将化合物 4 (1.37 g, $4.00 \mathrm{mmol})$ 悬浮于 $30 \mathrm{~mL}$ 四氢呋 喃及 $15 \mathrm{~mL}$ 水的混合溶剂中, 冰浴搅拌下少量多次加入 嗍氢化钠 $(0.91 \mathrm{~g}, 24.00 \mathrm{mmol}), 3.5 \mathrm{~h}$ 后经 TLC [V(石油 醚) $: V($ 乙酸乙酯 $)=6 ： 1]$ 检测显示反应完全. 向反应体 系中加入饱和氯化铵终止反应, 乙酸乙酯萃取 (100 mL × 2), 合并有机相, 饱和食盐水 $100 \mathrm{~mL}$ 洗涤, 无水硫酸钠干燥. 减压浓缩得粗品, 粗品经石油醚-乙 酸乙酯重结晶, 过滤, 干燥得白色固体 $51.04 \mathrm{~g}$, 收率 91\%. m.p. 137 $139{ }^{\circ} \mathrm{C} ;{ }^{1} \mathrm{H}$ NMR (400 MHz, $\left.\mathrm{CDCl}_{3}\right) \delta$ : 7.35 (d, $J=8.5 \mathrm{~Hz}, 2 \mathrm{H}), 6.92$ (d, $J=8.5 \mathrm{~Hz}, 2 \mathrm{H}), 6.12$ (d, $J=2.1 \mathrm{~Hz}, 1 \mathrm{H}), 6.01(\mathrm{~d}, J=2.1 \mathrm{~Hz}, 1 \mathrm{H}), 5.02 \sim 4.85(\mathrm{~m}$, $2 \mathrm{H}), 3.83(\mathrm{~s}, 3 \mathrm{H}), 3.73(\mathrm{~s}, 3 \mathrm{H}), 2.79 \sim 2.61(\mathrm{~m}, 2 \mathrm{H}), 2.26 \sim$ $2.14(\mathrm{~m}, 1 \mathrm{H}), 2.12 \sim 1.97(\mathrm{~m}, 1 \mathrm{H}) ;{ }^{13} \mathrm{C} \mathrm{NMR}(150 \mathrm{MHz}$, $\left.\mathrm{CDCl}_{3}\right) \delta: 159.48,159.36,157.03,154.65,133.74,127.59$, 114.09, 101.96, 94.81, 94.48, 55.48, 55.44, 29.37, 19.26; HRMS calcd for $\mathrm{C}_{17} \mathrm{H}_{18} \mathrm{O}_{4} 286.1205$, found 286.1187.

3.2.45- $(\beta-2,3,4,6$ - 四苯甲酸酯- $D$ - 葡萄糖氧基) $-2,3-$ 二氢-2-(4-甲氧基苯基)-7-甲氧基苯并二氢吡喃(6)的 合成

将化合物 5 (0.77 g, $2.69 \mathrm{mmol})$, 化合物 8 (2.67 g, $4.05 \mathrm{mmol}$ )溶于氯仿 $50 \mathrm{~mL}$, 室温搅拌下依次加入四丁 基溴化铵 $(0.44 \mathrm{~g}, 1.36 \mathrm{mmol})$ 及饱和碳酸钾溶液 $50 \mathrm{~mL}$. 室温搅拌 $10 \mathrm{~min}$ 后, $45{ }^{\circ} \mathrm{C}$ 下加热反应 $18 \mathrm{~h}$, 经 TLC $[V($ 石油醚 $): V($ 乙酸乙酯 $)=4: 1]$ 检测显示反应完全.
分液，水相用二氯甲烷萃取 $(40 \mathrm{~mL} \times 2)$ ，合并有机相， 饱和食盐水 $50 \mathrm{~mL}$ 洗涤, 无水硫酸钠干燥. 减压浓缩柱 层析得白色固体 $61.53 \mathrm{~g}$, 收率 66\%. m.p. 198 199 ${ }^{\circ} \mathrm{C}$; $[\alpha]_{\mathrm{D}}^{25}-27.8\left(c 0.38, \mathrm{CHCl}_{3}\right) ;{ }^{1} \mathrm{H} \mathrm{NMR}\left(400 \mathrm{MHz}, \mathrm{CDCl}_{3}\right)$ $\delta$ : $8.05(\mathrm{~d}, J=7.7 \mathrm{~Hz}, 2 \mathrm{H}), 7.98 \sim 7.93(\mathrm{~m}, 4 \mathrm{H}), 7.87$ (d, $J=7.7 \mathrm{~Hz}, 2 \mathrm{H}), 7.57 \sim 7.23(\mathrm{~m}, 14 \mathrm{H}), 6.91 \sim 6.83(\mathrm{~m}, 2 \mathrm{H})$, $6.35(\mathrm{~s}, 1 \mathrm{H}), 6.18(\mathrm{~s}, 1 \mathrm{H}), 6.05 \sim 5.96(\mathrm{~m}, 1 \mathrm{H}), 5.85(\mathrm{t}, J=$ $8.7 \mathrm{~Hz}, 1 \mathrm{H}), 5.73$ (t, $J=8.2 \mathrm{~Hz}, 1 \mathrm{H}), 5.39$ (t, $J=7.5 \mathrm{~Hz}$, $1 \mathrm{H}), 4.83 \sim 4.65(\mathrm{~m}, 2 \mathrm{H}), 4.56 \sim 4.44(\mathrm{~m}, 1 \mathrm{H}), 4.36(\mathrm{~s}$, $1 \mathrm{H}), 3.80$ (s, 3H), 3.64 (s, 3H), 2.75 (d, $J=16.8 \mathrm{~Hz}, 0.5 \mathrm{H})$, $2.65 \sim 2.53(\mathrm{~m}, 0.5 \mathrm{H}), 2.45(\mathrm{~d}, J=13.1 \mathrm{~Hz}, 0.5 \mathrm{H}), 2.35 \sim$ $2.21(\mathrm{~m}, 0.5 \mathrm{H}), 1.91(\mathrm{~s}, 1.5 \mathrm{H}), 1.75 \sim 1.66(\mathrm{~m}, 0.5 \mathrm{H}) ;{ }^{13} \mathrm{C}$ NMR (150 MHz, $\left.\mathrm{CDCl}_{3}\right) \delta: 166.32,165.91,165.40$, $165.27,165.17,159.47,159.44,159.30,156.78,156.70$, $155.94,155.83,133.72,133.66,133.60,133.54,133.50$, $133.25,130.04,129.95,129.86,129.62,129.30,129.23$, $128.86,128.78,128.62,128.50,127.56,127.51,114.03$, $105.13,99.78,99.57,96.13,95.63,95.57,77.73,72.88$, 72.82, 71.70, 71.64, 69.66, 63.44, 55.45, 29.10, 19.57, 19.40; HRMS calcd for $\mathrm{C}_{51} \mathrm{H}_{45} \mathrm{O}_{13}$ 865.2860, found 865.2847 .

3.2.5 5- $O-(\beta-2,3,4,6$ - 四苯甲酸酯- $D$ - 葡萄糖) $-7,4$ '- 二 甲氧基洋芹素(7)的合成

将化合物 6 (0.66 g, $0.77 \mathrm{mmol})$ 溶于二氯甲烷 60 $\mathrm{mL}$, 室温摚拌下加入 DDQ (0.53 g, $2.31 \mathrm{mmol}) .18 \mathrm{~h}$ 后, 经 TLC $[V$ (石油醚)： $V$ (乙酸乙酯 $)=4 ： 1]$ 检测显示反应 完全. 过滤, 二氯甲烷洗涤滤饼, 有机相用饱和食盐水 $50 \mathrm{~mL}$ 洗涤, 无水硫酸钠干燥. 减压浓缩柱层析得淡黄 色固体 $70.53 \mathrm{~g}$, 收率 78\%. m.p. 131 132 ${ }^{\circ} \mathrm{C}$; $[\alpha]_{\mathrm{D}}^{25}$ $-32.8\left(c \quad 0.35, \mathrm{CHCl}_{3}\right)$; ${ }^{1} \mathrm{H}$ NMR $\left(400 \mathrm{MHz}, \mathrm{CDCl}_{3}\right) \delta$ : $8.03 \sim 7.89(\mathrm{~m}, 8 \mathrm{H}), 7.74(\mathrm{~d}, J=8.6 \mathrm{~Hz}, 2 \mathrm{H}), 7.54 \sim 7.29$ $(\mathrm{m}, 12 \mathrm{H}), 6.98(\mathrm{~d}, J=8.6 \mathrm{~Hz}, 2 \mathrm{H}), 6.71(\mathrm{~s}, 1 \mathrm{H}), 6.58(\mathrm{~s}$, $1 \mathrm{H}), 6.39(\mathrm{~s}, 1 \mathrm{H}), 6.07 \sim 5.89(\mathrm{~m}, 2 \mathrm{H}), 5.81(\mathrm{t}, J=9.3 \mathrm{~Hz}$, $1 \mathrm{H}), 5.66(\mathrm{~d}, J=6.8 \mathrm{~Hz}, 1 \mathrm{H}), 4.68(\mathrm{~d}, J=11.7 \mathrm{~Hz}, 1 \mathrm{H})$, 4.49 (dd, $J=11.9,5.7 \mathrm{~Hz}, 1 \mathrm{H}), 4.32$ (s, 1H), 3.87 (s, 3H), $3.77(\mathrm{~s}, 3 \mathrm{H}) ;{ }^{13} \mathrm{C} \mathrm{NMR}\left(150 \mathrm{MHz}, \mathrm{CDCl}_{3}\right) \delta: 176.31$, $166.21,165.99,165.36,163.25,162.16,160.69,159.17$, $156.69,133.61,133.36,133.13,132.97,130.13,130.05$, $129.94,128.91,128.57,128.45,128.39,128.29,127.72$, $123.98,114.46,110.61,107.56,103.59,99.84,96.50$, 73.07, 72.93, 72.82, 69.62, 63.23, 55.85, 55.60; HRMS calcd for $\mathrm{C}_{51} \mathrm{H}_{41} \mathrm{O}_{14}$ 877.2496, found 877.2487.

3.2.67, $4^{\prime}$-二甲氧基洋芹素-5- $O$ - 葡萄糖苷(1)的合成

将化合物 7 (287 mg, $0.33 \mathrm{mmol}$ )溶于甲醇 $5 \mathrm{~mL}$ 、四 氢呋喃 $5 \mathrm{~mL}$ 以及水 $1 \mathrm{~mL}$ 的混合溶剂中, 室温摚拌下加 
入无水碳酸钾 $(90 \mathrm{mg}, 0.66 \mathrm{mmol})$. 室温搅拌反应 $5 \mathrm{~min}$ 后, 加热至 $45{ }^{\circ} \mathrm{C}$ 继续反应 $2 \mathrm{~h}$, 经 TLC [ $V$ (石油醚): $V($ 乙酸乙酯 $)=1: 4]$ 检测显示反应完全. 加入阳离子树 脂中和至中性, 过滤, 甲醇洗涤, 有机相减压浓缩得粗 品, 将所得粗品悬浮于 $10 \mathrm{~mL}$ 乙酸乙酯, 加热回流 30 $\min$ 后, 自然冷却, 过滤, 滤饼用乙酸乙酯洗涤, 真空干 燥得黄色固体 $1140 \mathrm{mg}$, 收率 93\%. m.p. 210 211 ${ }^{\circ} \mathrm{C}$; $[\alpha]_{\mathrm{D}}^{25}-40.5$ (c 0.45 , pyridine); ${ }^{1} \mathrm{H}$ NMR $(400 \mathrm{MHz}$, DMSO- $\left.d_{6}\right) \delta: 8.04$ (d, $\left.J=7.9 \mathrm{~Hz}, 2 \mathrm{H}, \mathrm{H}-2^{\prime}, 6^{\prime}\right), 7.11$ (d, $J=$ $\left.7.9 \mathrm{~Hz}, 2 \mathrm{H}, \mathrm{H}-3^{\prime}, 5^{\prime}\right), 7.10$ (s, H-8, 1H), 6.91 (s, H-6, 1H), 6.81 (s, H-3, 1H), 4.76 (d, $J=6.6 \mathrm{~Hz}, \mathrm{H}-1 ", 1 \mathrm{H}), 3.90$ (s, $\left.7-\mathrm{OCH}_{3}, 3 \mathrm{H}\right), 3.86\left(\mathrm{~s}, 3 \mathrm{H}, 4^{\prime}-\mathrm{OCH}_{3}\right), 3.75$ (dd, $J=12.0$, $5.0 \mathrm{~Hz}, 1 \mathrm{H}, \mathrm{H}-6 "), 3.52(\mathrm{~m}, \mathrm{H}-6 ", 1 \mathrm{H}), 3.50 \sim 3.16$ (m, 4H, H-2", 3", 4", 5"); ${ }^{13} \mathrm{C}$ NMR (150 MHz, DMSO- $\left.d_{6}\right) \delta$ : 176.95 (C-4), 163.61 (C-7), 162.10 (C-4'), 160.96 (C-2), 158.48 (C-9), 158.24 (C-5), 128.04 (C-2'), 122.75 (C-1'), 114.55 (C-3'), 109.30 (C-10), 106.49 (C-3), 104.18 (C-1"), 103.53 (C-6), 96.61 (C-8), 77.66 (C-3"), 75.77 (C-5"), 73.61 (C-2"), 69.95 (C-4"), 60.95 (C-6"), 56.09 (C-7OMe), 55.56 (C-4'-OMe); HRMS calcd for $\mathrm{C}_{23} \mathrm{H}_{25} \mathrm{O}_{10}$ 461.1448 , found 461.1439 .

\subsection{7 $1,2,3,4,6$-五苯甲酸酯- $D$ - 葡萄糖 $(\mathbf{1 0})$ 的合成}

将 $D$-葡萄糖 9 (9.00 g, $50.00 \mathrm{mmol})$ 溶于吡啶 150 $\mathrm{mL}$, 冰浴下缓慢滴加苯甲酰氯(43.70 mL, $375.00 \mathrm{mmol})$, 滴加完毕后撤去冰浴, 室温继续搅拌反应 $4 \mathrm{~h}$, 经 TLC $[V($ 石油醚 $): V($ 乙酸乙酯 $)=2: 1]$ 检测显示反应完全. 加入甲醇 $10 \mathrm{~mL}$ 终止反应, 减压蒸除大部分溶剂后将剩 余液倾入 $1 \mathrm{~mol} / \mathrm{L}$ 盐酸 $200 \mathrm{~mL}$ 中, 二氯甲烷萃取(100 $\mathrm{mL} \times 2)$, 合并有机相, 饱和食盐水 $100 \mathrm{~mL}$ 洗涤, 无水 硫酸钠干燥.减压除去溶剂, 95\%乙醇重结晶得白色粉末 $1029.46 \mathrm{~g}$, 收率 $84 \%$.

3.2 .81 -溴- $2,3,4,6$-四苯甲酸酯- $D$-葡萄糖 $(8)$ 的合成

将化合物 10 (7.00 g, $10.00 \mathrm{mmol}$ )溶于冰醋酸 100 $\mathrm{mL}$, 室温氮气保护下依次加入乙酰溴 $(3.70 \mathrm{~mL}, 50.00$ $\mathrm{mmol})$ 及甲醇 $(1.20 \mathrm{~mL}, 30.00 \mathrm{mmol})$, 室温搅拌 $8 \mathrm{~h}$, 经 TLC $[(V$ 石油醚 $): V($ 乙酸乙酯 $)=6: 1]$ 检测显示反应完 全. 向反应体系中加入二氯甲烷 $300 \mathrm{~mL}$ 稀释, 分别用 冷水、饱和碳酸氢钠水溶液、水各 $200 \mathrm{~mL}$ 洗涤, 无水 硫酸钠干燥, 减压浓缩柱层析得白色固体 $85.81 \mathrm{~g}$, 收 率 88\%. m.p. $129 \sim 130{ }^{\circ} \mathrm{C}$; $[\alpha]_{\mathrm{D}}^{25}-28.1$ (c 0.45, $\mathrm{CHCl}_{3}$ ); ${ }^{1} \mathrm{H}$ NMR (400 MHz, $\left.\mathrm{CDCl}_{3}\right) \delta: 8.07(\mathrm{~d}, J=7.4 \mathrm{~Hz}, 2 \mathrm{H})$, 8.00 (d, $J=7.4 \mathrm{~Hz}, 2 \mathrm{H}), 7.96$ (d, $J=7.6 \mathrm{~Hz}, 2 \mathrm{H}), 7.88$ (d, $J=7.5 \mathrm{~Hz}, 2 \mathrm{H}), 7.62 \sim 7.49(\mathrm{~m}, 3 \mathrm{H}), 7.47 \sim 7.36(\mathrm{~m}, 7 \mathrm{H})$, $7.33 \sim 7.29(\mathrm{~m}, 2 \mathrm{H}), 6.87(\mathrm{~s}, \mathrm{H}-1,1 \mathrm{H}), 6.27(\mathrm{t}, J=10.0 \mathrm{~Hz}$,
H-4, 1H), 5.82 (t, $J=10.1 \mathrm{~Hz}, \mathrm{H}-3,1 \mathrm{H}), 5.33$ (d, $J=10.4$ $\mathrm{Hz}, \mathrm{H}-2,1 \mathrm{H}), 4.74 \sim 4.65(\mathrm{~m}, 2 \mathrm{H}), 4.51(\mathrm{~d}, J=12.3 \mathrm{~Hz}$, $1 \mathrm{H})$; HRMS calcd for $\mathrm{C}_{41} \mathrm{H}_{33} \mathrm{O}_{11}$ 701.2023, found 701.2019 .

辅助材料(Supporting Information) 中间体及终产物 的 ${ }^{1} \mathrm{H}$ NMR 及 ${ }^{13} \mathrm{C}$ NMR 谱图. 这些材料可以免费从本刊 网站(http://sioc-journal.cn/)上下载.

\section{References}

[1] (a) Xu, W. N.; Gao, X. X.; Guo, X. L.; Chen, Y. C.; Zhang, W. M.; Luo, Y. S. J. Chin. Med. Mater. 2010, 33, 1736 (in Chinese). (徐维娜, 高晓霞, 郭晓玲, 陈玉婵, 章卫民, 罗源生, 中药材, 2010, 33, 1736.)

(b) Wang, H. G.; Zhou, M. H.; Lu, J. J.; Yu, B. Y. Chem. Ind. For Prod. 2008, 28, 1 (in Chinese).

(王红刚, 周敏华, 路晶晶, 余伯阳, 林产化学与工业, 2008, 28, 1.) (c) Men, W. L.; Lin, F.; Zuo, W. J.; Wang, H.; Dai, H. F. Chin. J. Nat. Med. 2012, 10, 234.

[2] (a) Liu, J.; Mei, W. L.; Cui, H. B.; Wu, J.; Dai, H. F. J. Chin. Med. Mater. 2008, 31, 340 (in Chinese).

(刘俊, 梅文莉, 崔海滨, 吴娇, 戴好富, 中药材, 2008, 31,340.) (b) Li, H. H.; Zhang, W. M.; Gao, X. X.; Wu, G. Q.; Chen, Y. C.; Wang, L. Chin. J. Exp. Trad. Med. Formul. 2011, 17, 100 (in Chinese). (李浩华，章卫民，高晓霞，吴关庆，陈玉婵，王否，中国实验方 剂学杂志, 2011, 17, 100.)

[3] (a) Zhou, M. H.; Wang, H. G.; Suolangjiba; Kou, J. P.; Yu, B. Y. J. Ethnopharmacol. 2008, 117, 345 .

(b) Qi, J.; Lu, J. J.; Liu, J. H.; Yu, B. Y. Chem. Pharm. Bull. 2009, 57,134

[4] Hara, H.; Ise, Y.; Morimoto, N.; Shimazawa, M.; Ichihashi, K.; Ohyama, M.; Iinuma, M. Biosci. Biotechnol. Biochem. 2008, 72, 335 .

[5] Jiang, S.; Jiang, Y.; Guan, Y. F.; Tu, P. F.; Wang, K. Y.; Chen, J. M. J. Chin. Pharm. Sci. 2011, 20, 609.

[6] (a) Hung, H. Y.; Qian, K. D.; Morris-Natschke, S. L.; Hsu, C. S.; Lee, K. H. Nat. Prod. Rep. 2012, 29, 580.

(b) Veitch, N. C.; Grayer, R. J. Nat. Prod. Rep. 2011, 28, 1626.

(c) Veitch, N. C.; Grayer, R. J. Nat. Prod. Rep. 2008, 25, 555.

(d) Williams, C. A.; Grayer, R. J. Nat. Prod. Rep. 2004, 21, 539

(e) Harborne, J. B.; Williams, C. A. Nat. Prod. Rep. 2001, 18, 310. (f) Harborne, J. B.; Williams, C. A. Nat. Prod. Rep. 1998, 15, 631.

[7] Chen, D.; Bi, D.; Song, Y. L.; Tu, P. F. Chin. J. Nat. Med. 2012, 10, 287.

[8] (a) Yang, W. Z.; Li, R. Y.; Han, W.; Zhang, W. D.; Sun, J. S. Chin. J. Org. Chem. 2012, 32, 1067 (in Chinese).

(杨为准, 李荣耀, 韩伟, 张卫东, 孙建松, 有机化学, 2012, 32, 1067.)

(b) Hu, Y.; Tu, Y. H.; Liu, D. Y.; Liao, J. X.; Sun, J. S. Org. Biomol. Chem. 2016, 14, 4842.

(c) Demetzos, C.; Skaltsounis, A. L.; Tillequin, F.; Koch, M. Carbohydr. Res. 1990, 207, 131.

(d) Kajjout, M.; Rolando, C. Tetrahedron 2011, 67, 4731.

(e) Alluis, B.; Dangles, O. Helv. Chim. Acta 2001, 84, 1133

(f) Midori, A. A.; Yumi, Y.; Masami, I. Org. Biomol. Chem. 2017, 15,5025 .

[9] Kang, M. M.; Ma, Z. L.; Liu, B.; Pan, D.; Li, J. Q. Chin. J. Org. Chem. 2017, 37, 1516 (in Chinese).

(康满满, 马志龙, 刘彪, 潘登, 李建其, 有机化学, 2017, 37, 1516.)

[10] Yan, S. Q.; Ren, S. M.; Ding, N.; Li, Y. X. Carbohydr. Res. 2018, 460,41 .

(Lu, Y.) 